\title{
Logic of prototypes and counterexamples: possibilities and limits
}

\author{
Thomas Vetterlein \\ Johannes Kepler University, Altenberger Straße 69, 4040 Linz, Austria
}

\begin{abstract}
Fuzzy sets are a popular tool to model vague properties. It is, however, well-known that this model usually involves a good degree of arbitrariness. In this contribution we consider the possibility of standardising the construction of fuzzy sets at least with regard to the borderline cases. To this end, we identify a vague property with the sets of clearly positive cases and clearly negative cases and under the assumption that the universe of discourse is a metric space, we determine the fuzzy set by what can be described as a linear interpolation. Following this idea, we discuss a number of logic-based approaches to reasoning under vagueness.
\end{abstract}

Keywords: Vague property, prototypical examples, counterexamples, logic of vagueness, Kleene logic.

\section{Introduction}

The discussion around the question how to choose a fuzzy set in order to represent a certain vague property in an appropriate way is probably as old as the theory of fuzzy sets itself. Let us review the common procedure. Assume that a set of worlds $W$ serves as the model of a situation that can vary in certain respects. We then say that a property $\varphi$ is vague if it is not for all $w \in W$ reasonably possible to $\operatorname{assign} \varphi$ one of the truth values "false" or "true". At certain $w \in W, \varphi$ applies undoubtedly; at certain $w \in W$ undoubtedly does not apply; but there are cases in which neither possibility can appropriately be assumed. Such cases are called borderline for $\varphi$. Modelling $\varphi$ by a fuzzy set means to define a map $u$ such that $u(w)=1$ or $u(w)=0$, respectively, in the clear cases, and $0<u(w)<1$ otherwise. The method is appealing; however, there is apparently no convincing way of justifying a particular choice of $u$. To specify the meaning of particular truth values is in fact a delicate matter and it is even more difficult to justify ways of defining operations on the set of truth values in order to interpret logical combinations of vague properties.

A discussion of this basic issue can be found, e.g., in [6]. Three primary ways of interpreting fuzzy sets are considered. According to one of these approaches, the notion of prototypicality for vague notions is central. In this case, the set of worlds $W$ is assumed to be endowed in some natural way with a similarity relation, such that each pair of two worlds is assigned a degree measuring their distinctness. Moreover, the question to which extent a world $w \in W$ is compatible with a property $\varphi$ is not decided independently for each $w$; instead, a subset $A$ of $W$ is chosen that is supposed to contain the clearly positive cases. The set $A$ in turn determines the fuzzy set modelling $\varphi$; the truth degree at a $w \in W$ is taken to be the supremum of the similarities between $w$ and the elements of $A$. Based on this idea, a number of formal approaches to dealing with vagueness have been defined. As an example, we may mention the paper [10].

The similarity-based approach to fuzzy sets is advantageous in several respects. First of all, it simplifies the choice of fuzzy sets, restricting the task to the decision about the clearly positive cases. Second, the interpretation of truth values is no longer a matter concerning each property separately; this matter is shifted to a more general level. It simply assumes that the set of worlds is actually a similarity space. In practice, the set of worlds might often be a subset of $\mathbb{R}^{n}$; in this case, it seems to be in fact reasonably possible to define a similarity relation, even though the choice will rarely be unique.

Here, we will elaborate on this idea, going one step further. Even if the similarity-based interpretation of fuzzy sets is convincing, there are practical disadvantages. The very reason of the problems caused by vagueness lies in the fact that we deal with different levels of granularities at the same time $[13,14]$. Vague properties refer to a coarse level of granularity; the chosen universe of discourse often refers to a finer, usually in fact to the finest possible level of granularity. Within the similarity-based approach, we can reasonably deal with two levels: one coarse level together with the level corresponding to our set of worlds.

It is, however, not easily possible to deal with levels of granularity in between the two extremes. Intuitively speaking, the graph of a fuzzy set determined by the prototypical cases by means of a similarity relation is always "equally steep". Consider, however, the following situation. Let us model the time of the day in a vague manner. Let $\alpha$ mean "around eleven o'clock" and let $\beta$ mean "around 11.02". Then we might take as the prototypical cases of $\alpha$ the time interval from, say, 10.50 to 11.10. In case of $\beta$ the interval will be narrower, say, the interval from 11.01 to 11.03 . How should we choose a similarity relation to obtain suitable fuzzy sets both for $\alpha$ and $\beta$ ? 
The task is impossible because $\beta$ refers to a finer level of granularity than $\alpha$. Considering a time difference of 30 minutes as similar to the degree $\frac{1}{2}$, we might arrive at a reasonable model for $\alpha$ : a fuzzy set whose support has, say, a width of around two hours. However, using the same similarity relation in case $\beta$ as well, we get a fuzzy set of nearly the same width. This is certainly counterintuitive.

In principle, there is an easy way to overcome this problem. Let us take into account not only the prototypical cases, but also the counterexamples, that is, the clearly negative cases. For $\alpha$, this is the time, say, before 10 and after 12 o'clock; for $\beta$, this is the time, say, before 10.58 and after 11.06. Furthermore, we may make use of the natural metric, the time difference, and construct the fuzzy sets in both cases by linear interpolation.

In the recent paper [12], we have applied this idea in the particular context of medical questionnaires. Such questionnaires consist of several items that patients are asked to evaluate; to this end they choose for each item a numerical degree indicating to which extent they think the item applies. The result is calculated from the patient's answers and is again a numerical degree. Identifying vague notions with pairs of prototypes and counterexamples proves in this context to be exactly the appropriate choice. However, the formalism is taylored to a quite specific application and the general case was not discussed.

It has been left open in [12] if there can be anything like a logic for prototypes and counterexamples. This is the issue of the present contribution. We surely do not offer definite solutions; the matter is somewhat tricky. Our intention is, at first place, to propagate the interpretation of fuzzy sets based on pairs of sets at all. Furthermore, our ultimate aim is to associate a logic with this idea, a logic which, if found satisfactory, could then reasonably be called a "logic of vagueness". In what follows, we consider a number of different possible approaches and discuss their advantages and disadvantages.

\section{Prototypes and counterexamples}

We will specify in this section the general framework that we propose to use in order to model vague properties.

A metric space is a pair $(W, d)$, where $W$ is a nonempty set and $d$ is a metric on $W$, that is, a mapping $d \in W \times W \rightarrow \mathbb{R}^{+}$such that, for any $u, v, w \in W$, (i) $d(v, w)=0$ if and only if $v=w$, (ii) $d(v, w)=$ $d(w, v)$, and (iii) $d(u, w) \leqslant d(u, v)+d(v, w)$.

The set $W$ will be our universe of discourse, commonly called the set of worlds. The metric $d$ on $W$ is intended to measure the distinctness of two worlds.

We note that, in principle, we could equally well work with a similarity relation, which in fact is more general and allows a greater flexibility. Roughly speaking, however, a metric often measures distances over the whole space and a similarity relation only in a restricted region around each point. This is in particular the case if the similarity relation is used for the construction of fuzzy sets, in the sense mentioned above. Hence we consider a metric as being more in line with our intentions. Apart from that, a typical case arising in praxis is the $\mathbb{R}^{n}$ endowed with the Euclidean metric.

We next consider the set of disjoint pairs of nonempty subsets of a metric space $(W, d)$ as well as the special fuzzy sets induced by them.

In what follows, we denote the distance of a point $a \in W$ from a non-empty subset $B$ of $W$ by $d$ as well; that is, we put $d(a, B)=\inf _{b \in B} d(a, b)$.

Definition 2.1 Let $(W, d)$ be a metric space. Then we call any pair $(A, B)$ of subsets of $W$ such that $A$ and $B$ are both non-empty and $A \cap B=\emptyset$ a contrasting pair in $W$. We denote the set of all contrasting pairs by $\mathcal{C}(W)$.

Moreover, for $(A, B) \in \mathcal{C}(W)$ we define

$$
s_{(A, B)}: W \rightarrow[0,1]: w \mapsto \frac{d(w, B)}{d(w, A)+d(w, B)},
$$

called the standard fuzzy set associated with $(A, B)$. We denote the set of all standard fuzzy sets by $\mathcal{F}(W)$.

Contrasting pairs are our intended models of vague properties. We note that pairs of disjoint (but otherwise arbitrary) subsets of a fixed set are called "orthopairs", e.g., in [2]. The first component is meant to represent the cases in which the property clearly holds, called prototypes; the second component is meant to represent the cases in which the property clearly does not hold, called counterexamples. Any remaining element is meant to represent a borderline case.

As we have mentioned right at the beginning of this contribution, models of vague properties typically involve arbitrariness. This is so for principal reasons. In fact, modelling a property referring to a coarse level of granularity within a framework that is based on a fine level of granularity implies the necessity to make the notion more specific that it actually is. This in turn is usually possible in more than one way. In order to model the vague notion "as is" we would have to choose a framework in line with its associated level of granularity. This is, in principle, well possible [14].

But also the present framework aims at combining several levels of granularity and is thus subjected to arbitrariness. The advantage compared to approaches based, e.g., on fuzzy sets without restriction of their shape is its minimality. We do not include more than what we consider as a basic requirement: determining the prototypes and counterexamples. We should certainly keep in mind that this simplification presumes that the underlying space is endowed with a metric in a natural way. 
The collection of pairs of disjoint subsets of a fixed set gives rise to a model that has been often considered in the literature. A detailed discussion of this approach can be found in $[7,8]$. For an overview of the different possible contexts see, e.g., [2, 3].

Here, the meaning of such pairs is restricted to the aspect of vagueness. As a consequence, we are led to a requirement that might distinguish our approach from others: the two sets that model a property must be non-empty. As we will see later, for the development of a formal framework this point is more than just a detail. We insist on it; we adopt in this contribution the following standpoint. Let $\varphi$ denote a possibly vague property, applicable to a certain sort of objects. Then $\varphi$ might be trivial, that is, always true or always false. In this case, $\varphi$ is not vague and if $\varphi$ is, e.g., always true, there are no counterexamples but any object is a prototype. The two trivial properties might be included in a model for practical reasons or not; let us disregard them for a moment and let $\varphi$ be a non-trivial property. By the very nature of what "property" means, there are objects to which $\varphi$ applies and objects to which $\varphi$ does not apply. Here, we argue with regard to the level of granularity to which $\varphi$ originally refers and this is in general a coarse level. But objects classified on the coarse level as fulfilling $\varphi$ correspond on the fine level to prototypes; and objects for which $\varphi$ is false correspond to counterexamples. In fact, objects considered on the coarse level are classified according to whether $\varphi$ holds or not; the coarse level is defined just in this way. It follows in particular that the set of prototypes and the set of counterexamples should both be chosen non-empty.

Given $(W, d)$, contrasting pairs and standard fuzzy sets are obviously in a one-to-one correspondence. Moreover, the definition of a fuzzy set out of a pair of subsets is done in line with the common procedure mentioned above: we first determine the prototypes $A$, then the counterexamples $B$, and we require that $u(w)=1$ exactly if $w \in A$, and $u(w)=0$ exactly if $w \in B$. If the universe is the real line, the borderline cases are moreover often assigned truth degrees by means of a simple linear interpolation. Definition 2.1 realises exactly this idea.

Within the present framework it is our concern to formalise the mutual relationships between vague properties and to design a logic capturing these interrelations. Accordingly, we are interested in the internal structure of the set of standard fuzzy sets $\mathcal{F}(W)$.

Most elementarily, we should establish a partial order corresponding to the expressive strength of properties. Let us see what we get if we do so in the standard way.

Let the set $\mathcal{F}(W)$ of standard fuzzy sets be endowed with the usual pointwise order. That is, for $s, t \in \mathcal{F}(W)$, we put $s \leqslant t$ if $s(w) \leqslant t(w)$ for all $w \in W$. Under the one-to-one correspondence be- tween $\mathcal{C}(W)$ and $\mathcal{F}(W)$, we partially order $\mathcal{C}(W)$ as well.

Lemma 2.2 For $\left(A^{+}, A^{-}\right),\left(B^{+}, B^{-}\right) \in \mathcal{C}(W)$, we have $\left(A^{+}, A^{-}\right) \leqslant\left(B^{+}, B^{-}\right)$if and only if $A^{+} \subseteq B^{+}$ and $A^{-} \supseteq B^{-}$.

Proof: Let $\left(A^{+}, A^{-}\right) \leqslant\left(B^{+}, B^{-}\right)$, that is, $s_{\left(A^{+}, A^{-}\right)} \leqslant s_{\left(B^{+}, B^{-}\right)}$. For $w \in A^{+}$we have $s_{\left(A^{+}, A^{-}\right)}(w)=1$, and $s_{\left(B^{+}, B^{-}\right)}(w)=1$ implies $w \in B^{+}$. Thus $A^{+} \subseteq B^{+}$, and similarly we see that $B^{-} \subseteq A^{-}$.

Conversely, let $A^{+} \subseteq B^{+}$and $B^{-} \subseteq A^{-}$. Then, for $w \in W, d\left(w, A^{+}\right) \geqslant d\left(w, B^{+}\right)$and $d\left(w, A^{-}\right) \leqslant$ $d\left(w, B^{-}\right)$and hence

$$
\begin{aligned}
s_{\left(A^{+}, A^{-}\right)}(w) & =\frac{1}{1+\frac{d\left(w, A^{+}\right)}{d\left(w, A^{-}\right)}} \\
& \leqslant \frac{1}{1+\frac{d\left(w, B^{+}\right)}{d\left(w, B^{-}\right)}}=s_{\left(B^{+}, B^{-}\right)}(w),
\end{aligned}
$$

that is, $\left(A^{+}, A^{-}\right) \leqslant\left(B^{+}, B^{-}\right)$.

We conclude that, if we want to build a logic based on the relative expressive strength only, we do not have to care about the underlying metric and in particular we do not need to take into account intermediate truth values.

Defining a logic for contrasting pairs turns out to be more involved than one could expect and we will indeed not consider anything else here. The difficulty about the present approach is implied by the following simple observation, which is a consequence of the fact that pairs in $\mathcal{C}(W)$ consist of non-empty sets.

Lemma 2.3 Elements $\left(A^{+}, A^{-}\right)$and $\left(B^{+}, B^{-}\right)$of $\mathcal{C}(W)$ have an infimum if and only if $A^{+} \cap B^{+} \neq \emptyset$. In this case,

$$
\left(A^{+}, A^{-}\right) \wedge\left(B^{+}, B^{-}\right)=\left(A^{+} \cap B^{+}, A^{-} \cup B^{-}\right) .
$$

Similarly, $\left(A^{+}, A^{-}\right)$and $\left(B^{+}, B^{-}\right)$have an supremum if and only if $A^{-} \cap B^{-} \neq \emptyset$. In this case,

$$
\left(A^{+}, A^{-}\right) \vee\left(B^{+}, B^{-}\right)=\left(A^{+} \cup B^{+}, A^{-} \cap B^{-}\right)
$$

Proof: If $A^{+} \cap B^{+} \neq \emptyset$, then clearly $\left(A^{+} \cap B^{+}, A^{-} \cup\right.$ $\left.B^{-}\right)$is the infimum of $\left(A^{+}, A^{-}\right)$and $\left(B^{+}, B^{-}\right)$. If $A^{+} \cap B^{+}=\emptyset$, then $\left(A^{+}, A^{-}\right)$and $\left(B^{+}, B^{-}\right)$do not have any lower bound and in particular no infimum.

Similarly we argue with regard to suprema.

In other words, $\mathcal{C}(W)$ is not a lattice. An immediate reaction to this deficiency could certainly be the call for a change of the definition of $\mathcal{C}(W)$; the requirement that both involved sets are non-empty could be dropped. Note, however, that we would be led in conflict with our intended interpretation. An element of $\mathcal{C}(W)$ is supposed to model a vague property; and a vague property necessarily has both prototypical examples and counterexamples. 
Having seen that the definition of a conjunction and disjunction causes problems in our framework, interpreting the negation need not be seen as difficult: the negation of a property can be modelled by the exchange of the set of prototypes with the set of counterexamples. Accordingly, we define

$$
\sim: \mathcal{C}(W) \rightarrow \mathcal{C}(W), \quad(A, B) \mapsto(B, A)
$$

Lemma 2.4 For any elements $(A, B),(C, D)$ of the poset $(\mathcal{C}(W) ; \leqslant)$, we have:

(C1) $(A, B) \leqslant(C, D)$ implies $\sim(C, D) \leqslant \sim(A, B)$,

$(\mathrm{C} 2) \sim \sim(A, B)=(A, B)$,

We proceed taking into account a first possibility how to realise in our framework also a conjunction and a disjunction.

\section{Adding propositions "false" and "true"}

The problem that infima in $\mathcal{C}(W)$ do not exist for all pairs can easily be solved: we add elements modelling falsity and truth. Namely, the proposition "false" is naturally modelled by the pair $(\emptyset, W)$, the proposition "true" by $(W, \emptyset)$.

Let us extend $\mathcal{C}(W)$ accordingly; let

$$
\overline{\mathcal{C}}(W)=\mathcal{C}(W) \cup\{(\emptyset, W),(W, \emptyset)\} .
$$

We extend the partial order on $\overline{\mathcal{C}}(W)$ such that $(\emptyset, W)$ is the bottom element and $(W, \emptyset)$ is the top element. We also extend $\sim$ to $\overline{\mathcal{C}}(W)$ in the expected way.

Lemma 3.1 Let $(W, d)$ be a metric space. Then $\overline{\mathcal{C}}(W)$ is a lattice. For $\left(A^{+}, A^{-}\right),\left(B^{+}, B^{-}\right) \in \mathcal{C}(W)$, we have

$$
\begin{aligned}
& \left(A^{+}, A^{-}\right) \wedge\left(B^{+}, B^{-}\right) \\
& = \begin{cases}\left(A^{+} \cap B^{+}, A^{-} \cup B^{-}\right) & \text {if } A^{+} \cap B^{+} \neq \emptyset, \\
(\emptyset, W) & \text { otherwise; }\end{cases} \\
& \left(A^{+}, A^{-}\right) \vee\left(B^{+}, B^{-}\right) \\
& = \begin{cases}\left(A^{+} \cup B^{+}, A^{-} \cap B^{-}\right) & \text {if } A^{-} \cap B^{-} \neq \emptyset, \\
(W, \emptyset) & \text { otherwise. }\end{cases}
\end{aligned}
$$

Proof: Again, in the case that $A^{+} \cap B^{+} \neq \emptyset$ it is clear that the infimum is as indicated. If $A^{+} \cap B^{+}=$ $\emptyset$, the only lower bound of $\left(A^{+}, A^{-}\right)$and $\left(B^{+}, B^{-}\right)$ is $(\emptyset, W)$, which is consequently the infimum.

The second equality is seen similarly.

Let us define the Logic of contrasting pairs, or LCP for short. We start with a countable set of variables $\varphi_{0}, \ldots$ and the constants $\perp, \top$. By a Boolean formula we mean a formula built up from the variables and constants by means of the binary connectives $\wedge, \vee$ and the unary connective $\neg$. Our syntactical objects are implications: pairs of Boolean formulas $\alpha$ and $\beta$, denoted by $\alpha \rightarrow \beta$.
We define the consequence relation on LCP semantically as follows. Let $(W, d)$ be a metric space. Then an evaluation in $(W, d)$ is a mapping $e$ from the Boolean formulas to $\overline{\mathcal{C}}(W)$ such that the following conditions are fulfilled: $e(\perp)=(\emptyset, W)$, $e(\top)=(W, \emptyset)$, and for Boolean formulas $\alpha, \beta$

$$
\begin{aligned}
e(\alpha \wedge \beta) & =e(\alpha) \wedge e(\beta), \\
e(\alpha \vee \beta) & =e(\alpha) \vee e(\beta), \\
e(\neg \alpha) & =\sim e(\alpha) .
\end{aligned}
$$

An implication $\alpha \rightarrow \beta$ is then said to be satisfied by $e$ if $e(\alpha) \leqslant e(\beta)$. A theory $\mathcal{T}$ is a set of implications; and $\mathcal{T}$ is said to semantically entail an implication $\zeta \rightarrow \eta$ in LCP if, whenever an evaluation $e$ satisfies all elements of $\mathcal{T}$, then $e$ satisfies $\zeta \rightarrow \eta$ as well.

We note that LCP can well be understood as a fuzzy logic - in the sense that fuzzy sets interpret the formulas. In fact, $\overline{\mathcal{C}}(W)$ is in a one-to-one correspondence with the set $\overline{\mathcal{F}}(W)$ consisting of the standard fuzzy sets as well as $\overline{0}$ and $\overline{1}$, the constant 0 and 1 mapping, respectively. Furthermore, the correspondence is an isomorphism of lattices. Thus LCP can alternatively be endowed with a semantics based on $\overline{\mathcal{F}}(W)$. The logic is certainly not truthfunctional. If some proposition $\alpha$ is interpreted by the fuzzy set $u$ and $\beta$ by the fuzzy set $v$, then $\alpha \wedge \beta$ is interpreted by the (pointwise) largest standard fuzzy set below $u$ and $v$ if there is any, otherwise by $\overline{0}$; similarly for $\alpha \vee \beta$; and $\neg \alpha$ is interpreted by the standard complement $W \rightarrow W, w \mapsto 1-u(w)$.

We might furthermore wonder if there is a logic that comes conceptually close to LCP but comprises an implication as a connective, along the lines of fuzzy logics or other substructural logics. Such a logic should be based on $\overline{\mathcal{C}}(W)$ endowed with the infimum as a conjunction and a further operation as an implication. Unfortunately, this idea is not straightforward to realise. There is no natural choice of an interpretation of the additional connective; a residual implication in $\overline{\mathcal{C}}(W)$ corresponding to the infimum does not exist.

In order to determine whether the logic LCP is in line with our intention of modelling the logical relationships between vague properties, let us have a closer look at the employed model, the structure $(\overline{\mathcal{C}}(W) ; \wedge, \vee, \sim, \mathbf{0}, \mathbf{1})$.

Proposition 3.2 Let $(W, d)$ be a metric space. Then $\overline{\mathcal{C}}(W)$ is a lattice with the bottom element $\mathbf{0}=(\emptyset, W)$ and the top element $\mathbf{1}=(W, \emptyset)$.

Moreover, the mapping

$$
\sim: \overline{\mathcal{C}}(W) \rightarrow \overline{\mathcal{C}}(W), \quad(A, B) \mapsto(B, A)
$$

is a complement function, that is, for any $(A, B),(C, D) \in \overline{\mathcal{C}}(W)$, properties $(\mathrm{C} 1),(\mathrm{C} 2)$ hold as well as the following one:

$\begin{aligned} \text { (C3) } & (A, B) \wedge \sim(A, B)=\mathbf{0} \text { and }(A, B) \vee \\ \sim(A, B)=\mathbf{1} & \end{aligned}$ 
By property (C3) of Proposition 3.2, we have that tertium non datur holds in LCP:

$$
\alpha \rightarrow \beta \vee \neg \beta
$$

is satisfied by any evaluation. We conclude that interpreting the conjunction and disjunction on purely order-theoretical grounds, we do not necessarily obtain a meaning in line with our intentions. In fact, let $(A, B) \in \overline{\mathcal{C}}(W)$. Then we have $(A, B) \vee(B, A)=\mathbf{1}$ and indeed, there is no property both above $(A, B)$ and above $(B, A)$ other than the property "true", even if $A \cup B$ is strictly contained in $W$. Consequently, the fact that prototypes and counterexamples do not necessarily exhaust all possibilities is not reflected in the logic LCP.

We finally note that an axiomatisation of LCP is not straightforward and not considered here. Notably, LCP is not just classical propositional logic. In fact, $\overline{\mathcal{C}}(W)$ is not a Boolean algebra. Distributivity does not in general hold. To see this, consider the case that $A^{+} \cap B^{+}=\emptyset$ and $A^{+} \cap C^{+} \neq \emptyset$; then

$$
\begin{aligned}
& \left(A^{+}, A^{-}\right) \wedge\left(\left(B^{+}, B^{-}\right) \vee\left(C^{+}, C^{-}\right)\right) \\
& =\left(A^{+} \cap\left(B^{+} \cup C^{+}\right), A^{-} \cup\left(B^{-} \cap C^{-}\right)\right) \\
& =\left(A^{+} \cup C^{+},\left(A^{-} \cup B^{-}\right) \cap\left(A^{-} \cup C^{-}\right)\right)
\end{aligned}
$$

need not coincide with

$$
\begin{aligned}
& \left(\left(A^{+}, A^{-}\right) \wedge\left(B^{+}, B^{-}\right)\right) \vee\left(\left(A^{+}, A^{-}\right) \wedge\left(C^{+}, C^{-}\right)\right) \\
& =\left(A^{+} \cap C^{+}, A^{-} \cup C^{-}\right) .
\end{aligned}
$$

\section{Kleene logic}

The setting defined in Section 2 comes close to the semantic framework of Kleene logic. We next rise the question whether this logic is suitable in the present context. We shall give a cautiously positive answer. Kleene logic has been the topic of much debate and to explain in a consistent manner what kind of statements this logic deals with is in fact not straightforward. Even more difficult is the situation for the closely related Belnap logic. See, e.g., $[4,15,5]$. Here, we consider a variant of Kleene logic based on implications as the basic syntactical objects; we then explain how we can "decompose" these implications to determine their meaning.

Also in this approach, we insist on our assumption that properties are modelled by pairs of non-empty subsets; but we require this interpretation for variables only.

Thus let us consider (a variant of) Kleene logic, denoted by $\mathrm{KL}$. We define the syntax in the same way as for LCP; that is, Boolean formulas and implications are defined in the same way as above.

Also the consequence relation in $\mathrm{KL}$ is defined similarly to LCP; however, the reference to a metric is dropped. An evaluation in a set $W$ is a mapping $e$ from the Boolean formulas to $P(W) \times P(W)$ such that $e(\perp)=(\emptyset, W), \quad e(\top)=(W, \emptyset)$, and for formulas $\alpha, \beta$ such that $e(\alpha)=\left(A^{+}, A^{-}\right)$and $e(\beta)=\left(B^{+}, B^{-}\right)$we have

$$
\begin{aligned}
e(\alpha \wedge \beta) & =\left(A^{+} \cap B^{+}, A^{-} \cup B^{-}\right), \\
e(\alpha \vee \beta) & =\left(A^{+} \cup B^{+}, A^{-} \cap B^{-}\right), \\
e(\neg \alpha) & =\left(A^{-}, A^{+}\right) .
\end{aligned}
$$

The notion of satisfaction of an implication as well as the semantic entailment in $\mathrm{KL}$ is defined similarly as in case of LCP.

In this case, an axiomatisation is possible along common lines. Call a sequent a pair consisting of a non-empty finite set of Boolean formulas and a single Boolean formula; we write

$$
\gamma_{1}, \ldots, \gamma_{k} \Rightarrow \delta
$$

A rule then consists of a finite, possibly empty, set of sequents called the assumptions and one further sequent called the conclusion.

Let us call KL the calculus whose rules are the following, for any sets of Boolean formulas $\Gamma$ and Boolean formulas $\alpha, \beta$ :

$$
\begin{aligned}
& \perp \Rightarrow \alpha \quad \alpha \Rightarrow \alpha \quad \alpha \Rightarrow \top \\
& \frac{\Gamma \Rightarrow \alpha \alpha \Rightarrow \beta}{\Gamma \Rightarrow \beta} \quad \frac{\Gamma \Rightarrow \alpha}{\Gamma, \beta \Rightarrow \alpha} \\
& \frac{\Gamma \Rightarrow \alpha \Gamma \Gamma \beta}{\Gamma \Rightarrow \alpha \wedge \beta} \quad \frac{\Gamma, \alpha, \beta \Rightarrow \gamma}{\Gamma, \alpha \wedge \beta \Rightarrow \gamma} \\
& \frac{\Gamma, \alpha \Rightarrow \gamma \quad \Gamma, \beta \Rightarrow \gamma}{\Gamma, \alpha \vee \beta \Rightarrow \gamma} \quad \frac{\Gamma \Rightarrow \alpha}{\Gamma \Rightarrow \alpha \vee \beta} \quad \frac{\Gamma \Rightarrow \beta}{\Gamma \Rightarrow \alpha \vee \beta} \\
& \frac{\alpha \Rightarrow \beta}{\neg \beta \Rightarrow \neg \alpha} \quad \frac{\neg \alpha \Rightarrow \beta}{\neg \beta \Rightarrow \alpha} \quad \frac{\alpha \Rightarrow \neg \beta}{\beta \Rightarrow \neg \alpha} \\
& \alpha, \neg \alpha \Rightarrow \beta \vee \neg \beta
\end{aligned}
$$

A proof of an implication $\zeta \rightarrow \eta$ from a theory $\mathcal{T}$ is defined as a tree of sequents in the expected way; in particular, a leaf may be of the form $\alpha \Rightarrow \beta$ if $\alpha \rightarrow \beta$ is in $\mathcal{T}$, and the root must be $\zeta \Rightarrow \eta$. Furthermore, a theory $\mathcal{T}$ is called consistent if $\mathcal{T}$ does not prove $\varphi \rightarrow \neg \varphi$ or $\neg \varphi \rightarrow \varphi$ for any variable $\varphi$. Then we have the following completeness theorem.

Theorem 4.1 Let $\mathcal{T}$ be a consistent theory and let $\zeta \rightarrow \eta$ be an implication. Then there is a proof of $\zeta \rightarrow \eta$ from $\mathcal{T}$ in $\mathbf{K L}$ if and only if $\mathcal{T}$ semantically entails $\zeta \rightarrow \eta$ in $\mathrm{KL}$.

Proof: (sketched) The soundness part is easily checked. To show completeness, assume that $\mathcal{T}$ does not prove $\zeta \rightarrow \eta$. On the set $\mathcal{B}$ of Boolean formulas, we define the equivalence $\approx$ requiring $\alpha \approx \beta$ if $\mathcal{T}$ proves $\alpha \rightarrow \beta$ and $\beta \rightarrow \alpha$. Then the operations $\wedge$, $\vee$, and $\neg$ are compatible with $\approx$, and the quotient $(\langle\mathcal{B}\rangle ; \wedge, \vee, \neg,\langle\perp\rangle,\langle\top\rangle)$ is a Kleene lattice. We have that $\mathcal{T}$ proves $\alpha \rightarrow \beta$ if and only if $\langle\alpha\rangle \leqslant\langle\beta\rangle$. As we may identify $\langle\mathcal{B}\rangle$ with a Kleene lattice of pairs of disjoint subsets of a set $W[9,11]$, some evaluation satisfies all elements of $\mathcal{T}$ but not $\zeta \rightarrow \eta$. 
The logic KL might be found appealing; however, for good reason, there has been a repeated debate around its proper interpretation. In the present context, it is well possible to provide a consistent interpretation: simply by going back to the semantics of the entailment relation. Note what this, regrettably, means: the pairs of subsets interpreting a compound formula have a quite technical character rather than providing a natural interpretation.

But we cannot expect anything else. As we noticed above, it is not reasonably possible to identify a compound formula with a property, which would be characterised by its prototypes and counterexamples. Instead, an implication involving compound formulas can be understood as a compound statement involving the prototypes and counterexamples of the variables. We may decompose an implication according to the definition of satisfaction.

For example, let $\varphi$ and $\psi$ variables and consider the implication

$$
\varphi \rightarrow \neg \psi .
$$

An implication reads both from left to right and from right to left; in fact (2) is equivalent to $\psi \rightarrow \neg \varphi$. In fact, (2) expresses, with regard to some interpretation in a set of worlds $W$ : if a world is among the prototypical examples of $\varphi$, then it is among the counterexamples of $\psi$; and if a world is a prototypical example of $\psi$, it is among the counterexamples of $\varphi$. Denoting the two sets of the pair interpreting some variable $\varphi$ by $[\varphi]^{+}$and $[\varphi]^{-}$, we have $[\varphi]^{+} \subseteq[\psi]^{-}$and $[\psi]^{+} \subseteq[\varphi]^{-}$.

To see the role of the binary connectives, consider the implication

$$
\neg(\varphi \wedge \neg \chi) \rightarrow \psi \wedge \omega .
$$

In a first step, we have to pull all the negations in front of the variables; and we have to consider the reversed implication as well. That is:

$$
\begin{aligned}
& \neg \varphi \vee \chi \rightarrow \psi \wedge \omega, \\
& \neg \psi \vee \neg \omega \rightarrow \varphi \wedge \neg \chi .
\end{aligned}
$$

That is, if a world $w$ is either in $[\varphi]^{-}$or $[\chi]^{+}$, then $w$ is both in $[\psi]^{+}$and $[\omega]^{+}$; and if $w$ is either in $[\omega]^{-}$ or in $[\psi]^{-}$, then $w$ is in $[\varphi]^{+}$and $[\chi]^{-}$.

Summarising, we may evaluate $\mathrm{KL}$ as follows. On the positive side, $\mathrm{KL}$ is based on a simple concept and its axiomatisation is feasible. The interpretation of the formulas of $\mathrm{KL}$ is moreover possible by direct reference to its semantic specification. On the negative side, this interpretation is cumbersome. The pair of subsets modelling a variable has a clear meaning; these sets contain the prototypes and counterexamples, respectively. But compound formulas seem not really to represent a property; the meaning of the modelling pair of sets is not so easily accessible. This also means that, in contrast to the previous logic LCP, an interpretation of $\mathrm{KL}$ on the basis of fuzzy sets does not seem to be possible.

\section{Further approaches}

We shall outline yet two more possible ways of defining a logical framework based on the idea that vague properties are modelled by their prototypes and counterexamples.

\subsection{Partial logics}

As a major problem about the present approach, we have initially noticed that the set $\mathcal{C}(W)$ of contrasting pairs in some metric space $W$ is not a lattice. The approach of Section 3 solves the issue by the addition of a bottom and a top element, which represent falsity and truth, respectively. An unpleasant consequence of this approach, however, is that the associated logic verifies the law of excluded middle; the disjunction of a proposition and its negation holds always true.

As a remedy, we could accept the fact that our framework does not always allow the combination of two properties in the sense of a conjunction or disjunction. If properties $\alpha, \beta$ are modelled by $(A, B),(C, D) \in \mathcal{C}(W)$, respectively, the pair $(A \cap C, B \cup D)$ well interprets $\alpha \wedge \beta$-but only if $A$ and $C$ are not disjoint. Similarly, $\alpha \vee \beta$ is appropriately interpreted by $(A \cup C, B \cap D)$-but only if $B$ and $D$ are not disjoint.

Taking this restriction seriously, we can define a partial logic, in which the formation of formulas is restricted; see, e.g., [1]. To this end, we introduce an additional connective $\diamond$ with the following meaning. For formulas $\alpha$ and $\beta, \alpha \diamond \beta$ holds if the sets of prototypes of $\alpha$ and $\beta$ have a non-empty intersection. That is, $\alpha$ being interpreted by $\left(A^{+}, A^{-}\right)$ and $\beta$ by $\left(B^{+}, B^{-}\right)$, we require $A^{+} \cap B^{+} \neq \emptyset$. The formation of the formula $\alpha \wedge \beta$ would then be bound to the condition $\alpha \diamond \beta$; similarly, the formula $\alpha \vee \beta$ would be allowed only under the condition $\neg \alpha \diamond \neg \beta$.

The approach is worth to be considered, it requires, however, a considerable change of viewpoint. In fact, whenever some properties $\alpha$ and $\beta$ are considered to be connected by a logical "and", it needs to be checked first if $\alpha$ does not contradict $\beta$. This restriction is, in principle, not unreasonable. For, in this case the sets of prototypes are disjoint and the formation of a logical conjunction is in fact unnecessary.

Moreover, following these lines, an interpretation in terms of fuzzy sets is possible in the same way as for the logic LCP. $\alpha$ being interpreted by the standard fuzzy set $u, \beta$ being interpreted by $v$, we interpret $\alpha \wedge \beta$ by the largest standard fuzzy set below $u$ and $v$, provided that $u$ and $v$ are lower bounded at all; otherwise, the combination would be undefined. 


\subsection{Independent treatment of the positive and negative cases}

Section 4 was devoted to (a modification of) Kleene logic, the logic KL. A drawback of $\mathrm{KL}$ is its cumbersome interpretation.

We may mention that there is a simple alternative to $\mathrm{KL}$. We understand $\mathrm{KL}$ as referring to the prototypes and counterexamples of properties. This idea can certainly be formalised also directly. We could allow explicit and separate reference to the prototypes and the counterexamples of some property.

This approach can be realised within a logic of distributive lattices. Specify a logic $\mathrm{DL}^{ \pm}$as follows. Let a set of symbols $\varphi_{0}, \ldots$ be given and for any such symbol $\varphi$, call $\varphi^{-}$and $\varphi^{+}$signed variables. Let a signed lattice formula be built up from the signed variables by means of the binary connectives $\wedge$ and $\vee$, and let an implication be an expression of the form $\alpha \rightarrow \beta$, where $\alpha$ and $\beta$ are signed lattice formulas.

On the semantic side, we fix a set of worlds $W$ and for each variable $\varphi$, we map $\varphi^{-}$and $\varphi^{+}$to disjoint non-empty subsets of $W$. Evaluations are defined on the basis of the set-theoretical operations and the satisfaction of an implication is defined by means of set-theoretical inclusion.

Compared to $\mathrm{KL}$, the difficulties of interpretation are overcome. $\mathrm{DL}^{ \pm}$is even more flexible than $\mathrm{KL}$ because it can refer to the positive cases separately from the negative ones. This, however, might in turn be found unsatisfactory; the implications $\alpha^{+} \rightarrow \beta^{+}$and $\beta^{-} \rightarrow \alpha^{-}$are unrelated. Moreover, an interpretation on the basis of fuzzy sets is not straightforward, simply because a fuzzy set models both prototypes and counterexamples of a property, but a signed variable refers only to one of these sets.

\section{Conclusion}

Specifying a vague property $\varphi$ within a model that is based on a finer level of granularity than $\varphi$ means, in a first step, delimiting those cases in which $\varphi$ is clearly true from those cases in which $\varphi$ is clearly wrong. The fuzzy sets that are used in practice often do not require more; the truth values of the borderline cases are calculated by means of a linear interpolation. In such a situation, reasoning about vague notions can be seen as reasoning about the clear cases. This point of view has been the basis of the present note.

Our intention has been to contribute to the often discussed problem what a "logic of vagueness" should look like. We have proposed to exploit the idea of modelling properties by what we call contrasting pairs, which are pairs of disjoint non-empty subsets of a metric space. Although this approach sounds easy, a realisation of a suitable logic leads to amazingly serious obstacles. We have discussed in total four possible logic-based approaches, in each case exhibiting advantages as well as drawbacks.

Our considerations might in fact imply that an ideal approach is not realistic. Nonetheless, all four mentioned approaches call for further elaborations and practical evaluations.

Relations to fuzzy logic exist for two of the approaches on the conceptual level. Technically, the considered logics do not fall into the framework of mathematical fuzzy logic, since truth-functionality is a missing feature. We do not view this as a significant problem though; the present contribution presumes that flexibility in this respect does not run counter to our general objective of exploring new frameworks for dealing with vagueness.

\section{References}

[1] S. Blamey, Partial logic, in: D. Gabbay, F. Guenthner (Eds.), "Handbook of philosophical logic. Vol. III: Alternatives to classical logic", D. Reidel Publishing Co., Dordrecht 1986; pp. $1-70$.

[2] D. Ciucci, D. Dubois, J. Lawry, Borderline vs. unknown: a comparison between three-valued valuations, partial models, and possibility distributions, in: Ll. Godo, H. Prade (Eds.), Working Papers of the ECAI-2012 Workshop on Weighted Logics for Artificial Intelligence (Montpellier, 28 Aug 2012), 2012; pp. 83 - 90.

[3] D. Ciucci, D. Dubois, J. Lawry, Borderline vs. unknown: comparing three-valued representations of imperfect information, Int. J. Approx. Reasoning 55 (2014), 1866 - 1889.

[4] D. Dubois, On ignorance and contradiction considered as truth-values, Log. J. IGPL $\mathbf{1 6}$ (2008), 195 - 216.

[5] D. Dubois, Author's response to Wansing and Belnap's generalized truth-values, Log. J. IGPL 18 (2010), 936 - 940.

[6] D. Dubois, H. Prade, The three semantics of fuzzy sets, Fuzzy Sets Syst. 90 (1997), 141 150.

[7] D. Dubois, H. Prade, An introduction to bipolar representations of information and preference, Int. J. Intell. Syst. 23 (2008), 866 - 877.

[8] D. Dubois, H. Prade, Gradualness, uncertainty and bipolarity: Making sense of fuzzy sets, Fuzzy Sets Syst. 192 (2012), 3 - 24.

[9] J. A. Kalman, Lattices with involution, Trans. Amer. Math. Soc. 87 (1958), 485 - 491.

[10] J. Lawry, T. Yongchuan, Uncertainty modelling for vague concepts: a prototype theory approach, Artif. Intell. 173 (2009), 1539 - 1558.

[11] A. P. Pynko, Implicational classes of De Morgan lattices, Discrete Math. 205 (1999), 171 181.

[12] T. Vetterlein, A. Zamansky, Reasoning with graded information: the case of diagnostic scales in healthcare, submitted. 
[13] T. Vetterlein, Vagueness - a mathematician's perspective, in: P. Cintula, C. Fermüller, Ll. Godo, P. Hájek (Eds.), "Understanding vagueness"; pp. 65 - 91.

[14] T. Vetterlein, Vagueness: where degree-based approaches are useful, and where we can do without, Soft Computing 16 (2012), 1833 1844.

[15] H. Wansing, N. Belnap, Generalized truth values. A reply to Dubois, Log. J. IGPL 18 (2010), $921-935$. 